\title{
A Method for Solving Hermitian Pentadiagonal Block Circulant Systems of Linear Equations
}

\author{
Borislav V. Minchev ${ }^{1}$ and Ivan G. Ivanov ${ }^{2}$ \\ 1 Department of Computer Science \\ University of Bergen, Norway \\ Borko.Minchev@ii.uib.no \\ http://www.ii.uib.no/ borko \\ ${ }^{2}$ Faculty of Economics and Business Administration \\ Sofia University, Sofia 1113, Bulgaria \\ i_ivanov@feb.uni-sofia.bg
}

\begin{abstract}
A new effective method and its two modifications for solving Hermitian pentadiagonal block circulant systems of linear equations are proposed. New algorithms based on the proposed method are constructed. Our algorithms are then compared with some classical techniques as far as implementation time is concerned, number of operations and storage. Numerical experiments corroborating the effectiveness of the proposed algorithms are also reported.
\end{abstract}

\section{Introduction}

Linear systems of equations having circulant coefficient matrices appear in many applications. For example in the finite difference approximation to an elliptic equations subject to periodic boundary conditions [2,8] and approximating periodic functions using splines $[1,9]$. In case when multidimensional problems are concerned the matrices of coefficients of the resulting linear systems are block circulant matrices [7].

In this paper we propose a new method and its two modifications for solving Hermitian pentadiagonal block circulant systems of linear equations. It is known that these systems have the form

$$
W x=f,
$$

where

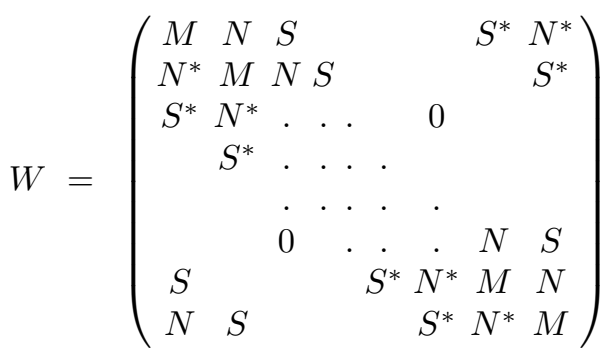


is a Hermitian pentadiagonal block circulant matrix with block size $n . M, N$ and $S$ are $m \times m$ matrices, $x=\left\{x_{i}\right\}_{i=1, \ldots, n}, f=\left\{f_{i}\right\}_{i=1, \ldots, n}$, are column vectors with block size $n, x_{i}$ and $f_{i}$, are blocks with size $m \times 1$.

Our goal is to construct a new effective method for solving of (1) and to compare it with some classical techniques.

The paper is organized as follows: in section 2 we present the new method and discuss its two modifications based on different approaches for using the Woodbury's formula [4]; in section 3 we report some numerical experiments corroborating the effectiveness of the proposed algorithms.

\section{A Modification of LU Factorizations}

Adapting the ideas suggested in [6], we construct a new method for solving linear systems with coefficient matrices of the form (2). Our approach is based on the solution of a special nonlinear matrix equation. One can fine the solution of (1) using the following steps:

Step 1. Solve the parametric linear system

$$
T y=f
$$

where

$$
T=\left(\begin{array}{ccccccc}
X & Y & S & & & \\
Y^{*} & Z & N & & & 0 & \\
S^{*} & N^{*} & M & \cdot & \cdot & & \\
& & \cdot & \cdot & \cdot & \cdot & S \\
& 0 & & \cdot & \cdot & \cdot & N \\
& & & & S^{*} & N^{*} & M
\end{array}\right)
$$

is pentadiagonal matrix with block size $n$. It has a block Toeplitz structure except for the north-western corner, $y=\left\{y_{i}\right\}_{i=1, \ldots, n}$, and $f=\left\{f_{i}\right\}_{i=1, \ldots, n}$ are column vectors with blocks size $n, y_{i}$ and $f_{i}$ are blocks with size $m \times 1$.

Matrix $T$ admits the following $\mathrm{LU}$ factorization

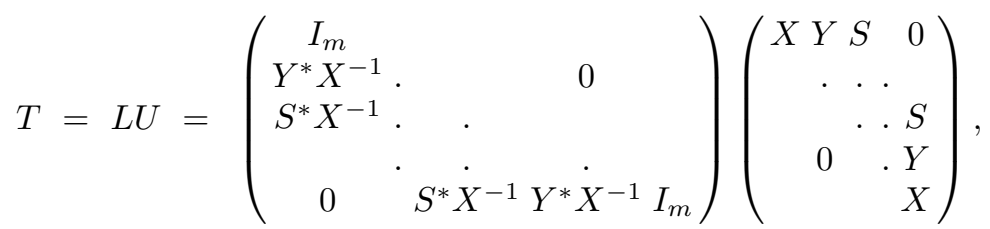

where $I_{m}$ is the identity matrix with size $m \times m$.

The above decomposition exists when the parameters $X=X^{*}, Y$ and $Z=Z^{*}$ satisfy the relations

$$
\mid \begin{aligned}
& Z=Y^{*} X^{-1} Y+X \\
& N=Y^{*} X^{-1} S+Y \\
& M=S^{*} X^{-1} S+Z
\end{aligned}
$$

Let us introduce the following notations

$$
F=\left(\begin{array}{cc}
X & Y \\
Y^{*} & Z
\end{array}\right), \quad Q=\left(\begin{array}{cc}
S & 0 \\
N & S
\end{array}\right), \quad R=\left(\begin{array}{cc}
M & N \\
N^{*} & M
\end{array}\right) .
$$


If $F$ is a positive definite solution of the matrix equation

$$
F+Q^{*} F^{-1} Q=R
$$

and $X=X^{*}>0, Z=Z^{*}>0$, then the blocks $X, Y$ and $Z$ satisfy the system (4).

Thus, solving the linear system (3) is equivalent to solving two simpler systems

$$
\begin{aligned}
& L z=f, \quad z=\left\{z_{i}\right\}_{i=1, \ldots, n} \\
& U y=z, \quad y=\left\{y_{i}\right\}_{i=1, \ldots, n} .
\end{aligned}
$$

Step 2. Solve the pentadiagonal block Toeplitz linear system

$$
P u=f
$$

where

$$
P=\left(\begin{array}{cccccccc}
M & N & S & & & & \\
N^{*} & M & N & & & & 0 & \\
S^{*} & N^{*} & M & \ldots & & & \\
& \cdot & \cdot & \cdots & . & & \\
& & & \cdots & \cdot & . & S \\
& 0 & & & \cdot & . & . & \\
& & & & S^{*} & N^{*} & M
\end{array}\right)
$$

is a Hermitian pentadiagonal block Toeplitz matrix with block size $n, u=$ $\left\{u_{i}\right\}_{i=1, \ldots, n}$ and $f=\left\{f_{i}\right\}_{i=1, \ldots, n}$ are column vectors with block size $n, u_{i}$ and $f_{i}$ are blocks with size $m \times 1$.

The matrices $T$ and $P$ satisfy the relation $P=T+J_{2} \hat{V}$, where

$$
J_{2}=\left(\begin{array}{ccccc}
I_{m} & 0 & 0 & \ldots & 0 \\
0 & I_{m} & 0 & \ldots & 0
\end{array}\right)^{T}, \quad \hat{V}=\left(\begin{array}{lllll}
M-X & N-Y & 0 & \ldots & 0 \\
N^{*}-Y^{*} & M-Z & 0 & \ldots & 0
\end{array}\right)
$$

are matrices with block size $n \times 2$ and $2 \times n$ respectively.

Using the Woodbury's formula we have

$$
P^{-1}=T^{-1}-T^{-1} J_{2}\left[I_{2 m}+\hat{V} T^{-1} J_{2}\right]^{-1} \hat{V} T^{-1},
$$

where $I_{2 m}$ is the identity matrix with size $2 m \times 2 m$.

For the solution $u$ of (7) we have

$$
u=P^{-1} f=y-T^{-1} J_{2}\left[I_{2 m}+\hat{V} T^{-1} J_{2}\right]^{-1} \hat{V} y .
$$

One can find the matrix $T^{-1} J_{2}$ by solving $2 m$ linear systems of type (3) with right-hand sides the corresponding to different columns of $J_{2}$. This approach does not take into consideration the very sparse nonzero structure of $J_{2}$. For real $M, N$ and $S$ it costs $O\left(20 \mathrm{~nm}^{3}\right)$ flops and needs to store $2 \mathrm{~nm}^{2}$ real numbers. In order to decrease the number of operation for computing $T^{-1} J_{2}$ we propose 
a new approach which is based on the ideas suggested in [5].

Let us denote the block columns vectors of $J_{2}$ with $E_{1}$ and $E_{2}$ respectively i.e.

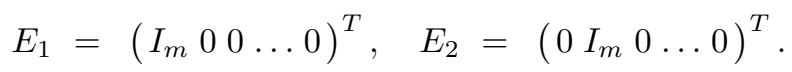

Put $A=Y^{*} X^{-1}$ and $B=S^{*} X^{-1}$.

The matrix $T$ admits the following decomposition

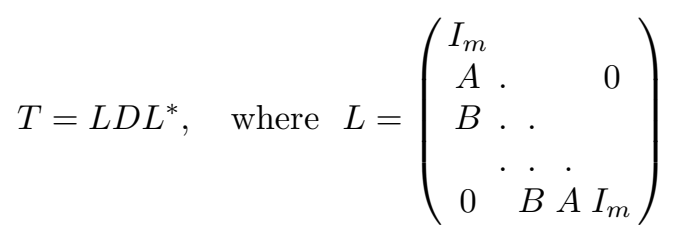

is a square matrix of block size $n$ and $D=\operatorname{diag}(X, \ldots, X)$.

Let $\left(L^{-1}\right)_{i j}$ be blocks of the matrix $L^{-1}$. We have

$$
\left(L^{-1}\right)_{i j}=\left\{\begin{array}{ll}
0 & i<j \\
Z_{i-j+1} & i \geq j,
\end{array} \text { where } \begin{array}{l}
Z_{1}=I_{m}, \quad Z_{2}=-A, \quad Z_{3}=A^{2}-B, \\
Z_{j}=-A Z_{j-1}-B Z_{j-2} \quad \text { for } j=4 \ldots n .
\end{array}\right.
$$

Obviously $D^{-1}=\operatorname{diag}\left(X^{-1}, \ldots, X^{-1}\right)$.

One can compute $T^{-1} J_{2}$ by consecutively calculation of $T^{-1} E_{1}$ and $T^{-1} E_{2}$ using the following algorithm:

Algorithm RP Recursively computation for Pentadiagonal system

- Find the cells $K_{i}=X^{-1} Z_{i} \quad$ for $i=1, \ldots, n$ by the formulas

$$
\begin{aligned}
& K_{1}=X^{-1} \\
& K_{2}=-X^{-1} A \\
& K_{i}=-K_{i-1} A-K_{i-2} B \quad \text { for } i=3, \ldots n .
\end{aligned}
$$

- Compute the blocks $\left(T^{-1} E_{1}\right)_{i}$ and $\left(T^{-1} E_{2}\right)_{i}$ by the formulas

$$
\begin{aligned}
\left(T^{-1} E_{1}\right)_{n} & =K_{n} \\
\left(T^{-1} E_{1}\right)_{n-1} & =K_{n-1}-A^{*}\left(T^{-1} E_{1}\right)_{n} \\
\left(T^{-1} E_{1}\right)_{i} & =K_{i}-A^{*}\left(T^{-1} E_{1}\right)_{i+1}-B^{*}\left(T^{-1} E_{1}\right)_{i+2} \quad \text { for } i=n-2, \ldots, 2 \\
\left(T^{-1} E_{1}\right)_{1} & =X^{-1}-A^{*}\left(T^{-1} E_{1}\right)_{2}-B^{*}\left(T^{-1} E_{1}\right)_{3} \\
\left(T^{-1} E_{2}\right)_{n} & =K_{n-1} \\
\left(T^{-1} E_{2}\right)_{n-1} & =K_{n-2}-A^{*}\left(T^{-1} E_{2}\right)_{n} \\
\left(T^{-1} E_{2}\right)_{i} & =K_{i-1}-A^{*}\left(T^{-1} E_{2}\right)_{i+1}-B^{*}\left(T^{-1} E_{2}\right)_{i+2} \text { for } i=n-2, \ldots, 2 \\
\left(T^{-1} E_{2}\right)_{1} & =-A^{*}\left(T^{-1} E_{2}\right)_{2}-B^{*}\left(T^{-1} E_{2}\right)_{3} .
\end{aligned}
$$

If the blocks $M, N$ and $S$ are real the algorithm RP costs $O\left(12 \mathrm{~nm}^{3}\right)$ flops and needs to store $(3 n+2) m^{2}$ real numbers; According to the above algorithm in the next step we consider two different approaches for solving (1). 
Step 3. Solve the system (1)

3.1 The matrix $W$ satisfies the relation

$$
W=P+\tilde{U} \tilde{V}
$$

where

$$
\tilde{U}=\left(\begin{array}{llll}
I_{m} & 0 & 0 & 0 \\
0 & I_{m} & 0 & 0 \\
\vdots & \vdots & \vdots & \vdots \\
0 & 0 & I_{m} & 0 \\
0 & 0 & 0 & I_{m}
\end{array}\right), \quad \tilde{V}=\left(\begin{array}{ccccc}
0 & 0 & \ldots & S^{*} & N^{*} \\
0 & 0 & \ldots & 0 & S^{*} \\
S & 0 & \ldots & 0 & 0 \\
N & S & \ldots & 0 & 0
\end{array}\right)
$$

are the matrices with block size $n \times 4$ and $4 \times n$ respectively.

Using the Woodbury's formula we have

$$
W^{-1}=P^{-1}-P^{-1} \tilde{U}\left[I_{4 m}+\tilde{V} P^{-1} \tilde{U}\right]^{-1} \tilde{V} P^{-1},
$$

where $I_{4 m}$ is the identity matrix with size $4 m \times 4 m$.

The solution $x$ of (1) is obtained from the vector $u$ as follows

$$
x=W^{-1} f=u-P^{-1} \tilde{U}\left[I_{4 m}+\tilde{V} P^{-1} \tilde{U}\right]^{-1} \tilde{V} u .
$$

Denote the block columns vectors of $\tilde{U}$ with $E_{1}, E_{2}, E_{n-1}$ and $E_{n}$ respectively. Computation of $P^{-1} \tilde{U}$ can be done by consecutively calculations of $P^{-1} E_{1}$, $P^{-1} E_{2}, P^{-1} E_{n-1}$ and $P^{-1} E_{n}$ using formula (9). For $\mathrm{i}=1,2, n-1, n$ we have

$$
P^{-1} E_{i}=T^{-1} E_{i}-T^{-1} J_{2}\left[I_{2 m}+\hat{V} T^{-1} J_{2}\right]^{-1} \hat{V} T^{-1} E_{i} .
$$

Numerical implementation of formulas (10) is very "cheap" since we already know from Step 2 the elements $T^{-1} J_{2}$ and $\left[I_{2 m}+\hat{V} T^{-1} J_{2}\right]^{-1}$. We recommend formulas (10) instead of solving $4 m$ linear system of the form (7) with right hand side the corresponding column vectors of $\tilde{U}$. It is easy to observe that the blocks of $T^{-1} E_{n-1}$ and $T^{-1} E_{n}$ satisfy the relations

$$
\begin{aligned}
\left(T^{-1} E_{n-1}\right)_{n} & =K_{2}^{*}, \\
\left(T^{-1} E_{n-1}\right)_{i} & =K_{n-i}^{*}-K_{n-i+1}^{*} A \quad \text { for } i=n-1, \ldots, 1, \\
\left(T^{-1} E_{n}\right)_{i} & =K_{n+1-i}^{*} \quad \text { for } i=n, \ldots, 1,
\end{aligned}
$$

where $K_{i}$ for $i=1, \ldots, n$ are the blocks from algorithm RP.

3.2 In order to decrease the size of the inverse matrix in Woodbury's formula we propose the following decomposition of matrix $W$

$$
W=\left(\begin{array}{cc}
P & V \\
V^{*} & R
\end{array}\right),
$$


where $P$ is from (8), with block size $n-2 \times n-2, R$ is from (5) and

$$
V^{*}=\left(\begin{array}{ccccc}
S & 0 & \ldots & S^{*} & N^{*} \\
N & S & \ldots & 0 & S^{*}
\end{array}\right)
$$

is a matrix with block size $2 \times n-2$.

Put

$$
\begin{gathered}
\hat{x}=\left(x_{1}, \ldots, x_{n-2}\right)^{T}, \quad \tilde{x}=\left(x_{n-1} x_{n}\right)^{T}, x=\left(\begin{array}{c}
\hat{x} \\
\tilde{x}
\end{array}\right), \\
\hat{f}=\left(f_{1}, \ldots, f_{n-2}\right)^{T}, \quad \tilde{f}=\left(f_{n-1} f_{n}\right)^{T}, f=\left(\begin{array}{c}
\hat{f} \\
\tilde{f}
\end{array}\right) .
\end{gathered}
$$

In this notation system (1) can be written in the form

$$
\left(\begin{array}{ll}
P & V \\
V^{*} & R
\end{array}\right)\left(\begin{array}{l}
\hat{x} \\
\tilde{x}
\end{array}\right)=\left(\begin{array}{l}
\hat{f} \\
\tilde{f}
\end{array}\right),
$$

which is equivalent to

$$
\mid \begin{aligned}
& G \hat{x}=r \\
& \tilde{x}=R^{-1}\left(\tilde{f}-V^{*} \hat{x}\right),
\end{aligned}
$$

where $G=P-V R^{-1} V^{*}, \quad r=\hat{f}-V R^{-1} \tilde{f}$.

By Woodbury's formula we have

$$
G^{-1}=P^{-1}+P^{-1} V\left[R-V^{*} P^{-1} V\right]^{-1} V^{*} P^{-1} .
$$

Then

$$
\hat{x}=G^{-1} r=z+P^{-1} V\left[R-V^{*} P^{-1} V\right]^{-1} V^{*} z,
$$

where $z=P^{-1} r$ can be computed by means of Step 2 .

Denote the block columns vectors of $V$ with $H_{1}$ and $H_{2}$ respectively. Computation of $P^{-1} V$ can be done by consecutively calculations of $P^{-1} H_{1}$ and $P^{-1} H_{2}$ using formula (9). For $\mathrm{i}=1,2$

$$
P^{-1} H_{i}=T^{-1} H_{i}-T^{-1} J_{2}\left[I_{2 m}+\hat{V} T^{-1} J_{2}\right]^{-1} \hat{V} T^{-1} H_{i}
$$

Numerical implementation of the last formulas is again "cheap" since we already know from Step 2 the elements $T^{-1} J_{2}$ and $\left[I_{2 m}+\hat{V} T^{-1} J_{2}\right]^{-1}$. The blocks of $T^{-1} H_{1}$ and $T^{-1} H_{2}$ satisfy the relations

$$
\begin{aligned}
\left(T^{-1} H_{1}\right)_{i} & =\left(T^{-1} E_{1}\right)_{i} S^{*}+K_{n-2-i}^{*} S+K_{n-i-1}^{*} \tilde{Q} \quad \text { for } i=1, \ldots, n-3 \\
\left(T^{-1} H_{1}\right)_{n-2} & =\left(T^{-1} E_{1}\right)_{n-2} S^{*}+K_{1}^{*} \tilde{Q} \\
\left(T^{-1} H_{2}\right)_{i} & =\left(T^{-1} E_{1}\right)_{i} N^{*}+\left(T^{-1} E_{2}\right)_{i} S^{*}+K_{n-1-i}^{*} S \quad \text { for } i=1, \ldots, n-2,
\end{aligned}
$$

where $\tilde{Q}=N-A S$ and $K_{i}$ for $i=1, \ldots, n$ are the blocks from algorithm RP. 


\section{$3 \quad$ Numerical Experiments}

In this section we compare our algorithms with some classical techniques for solving (1), with $W$ given as in (2), and the exact solution $x=(1,1, \ldots, 1)^{T}$.

In our numerical experiments, $W$ is Hermitian Pentadiagonal Block Circulant, with several block size $n$. The algorithms are compared by means of execution times and accuracy of the solution.

The codes are written in MATLAB language and computations are done on an AMD computer. The results of the experiments are given in different tables for each example.

The following notations are used: $L U$ stands for classical $L U$ factorization; CHOL stands for the classical Cholesky factorization; M_RP(4m) stands for algorithm based on the proposed new method using step 3.1; M_RP $(2 \mathrm{~m})$ stands for algorithm based on the proposed new method using step 3.2; Err. $=\| x-$ $\tilde{\tilde{x}} \|_{\infty}$, where $\tilde{\tilde{x}}$ is the computed solution.

To solve the system (1) we need to compute a positive definite solution of the matrix equation (6). The sufficient condition for the existence of a positive definite solution is $\left\|R^{-\frac{1}{2}} Q R^{-\frac{1}{2}}\right\| \leq \frac{1}{2}$, (see [3]). The cells of the matrix $W$ in next two examples which form matrices $R$ and $Q$ satisfy this condition.

Example 1. Let

$$
\begin{gathered}
M=\left(\begin{array}{ccc}
8 & 1-i & 1.5 \\
1+i & 9 & 1 \\
1.5 & 1 & 8
\end{array}\right), N=\left(\begin{array}{ccc}
0 & 1 & 0 \\
0 & 2 & 0 \\
1-i & 0 & 0
\end{array}\right) \\
S=\left(\begin{array}{ccc}
1.2-3 i & -0.3-i & 0.1 \\
-0.30 & 2.1 & 0.2 \\
0.1 & 0.2 & 0.65+2 i
\end{array}\right)
\end{gathered}
$$

We present the execution time (in seconds) and the error, of each algorithm for different values of $n$ in the table.

Example 2. Let

$$
M=\operatorname{circ}(22,-8,1, \ldots, 1,-8), \quad N=\operatorname{circ}(-7.2,1.8, \ldots, 1.8)
$$

are circulant matrices and $S=I$.

\section{Conclusions}

The proposed new algorithms M_RP $(2 \mathrm{~m})$ and M_RP $(4 \mathrm{~m})$ are faster than the classical $L U$ and $C H O L$. Theoretical investigation and numerical experiments suggest that algorithm M_RP $(2 \mathrm{~m})$ is most suitable for implementation. This is due to the size of the inverse matrix in Woodbury's formula. The inverse matrix in M_RP $(2 \mathrm{~m})$ is twice smaller than the inverse matrix in M_RP $(4 \mathrm{~m})$. This leads to the considerable decrease of the execution time. 
Table 1. Execution time (in seconds) and errors

\begin{tabular}{||l||c|c||c|c||c|c||}
\hline \hline \multirow{2}{*}{ Algorithm } & $n=4000$ & \multicolumn{2}{c||}{$n=6000$} & \multicolumn{2}{|c||}{$n=8000$} \\
\cline { 2 - 7 } & Err. & time & Err. & time & Err. & time \\
\hline \multicolumn{7}{|c||}{ Example $1, m=3$} \\
\hline LU & $1.4482 \mathrm{e}-015$ & 2.95 & $1.4482 \mathrm{e}-015$ & 6.04 & $1.4482 \mathrm{e}-015$ & 10.15 \\
\hline CHOL & $2.2888 \mathrm{e}-015$ & 2.00 & $2.2888 \mathrm{e}-015$ & 3.23 & $2.2888 \mathrm{e}-015$ & 5.44 \\
\hline M_RP $(4 \mathrm{~m})$ & $4.2635 \mathrm{e}-014$ & 1.95 & $4.1064 \mathrm{e}-014$ & 3.14 & $4.7126 \mathrm{e}-014$ & 5.02 \\
\hline M_RP $(2 \mathrm{~m})$ & $3.3956 \mathrm{e}-014$ & 1.63 & $4.1081 \mathrm{e}-014$ & 2.62 & $6.0280 \mathrm{e}-014$ & 4.34 \\
\hline \hline \multicolumn{7}{|c||}{ Example $2, m=7$} \\
\hline LU & $1.7764 \mathrm{e}-015$ & 3.49 & $1.7764 \mathrm{e}-015$ & 6.66 & $1.7764 \mathrm{e}-015$ & 11.01 \\
\hline CHOL & $1.9984 \mathrm{e}-015$ & 2.39 & $1.9984 \mathrm{e}-015$ & 3.90 & $1.9984 \mathrm{e}-015$ & 5.98 \\
\hline M_RP $(4 \mathrm{~m})$ & $2.6182 \mathrm{e}-014$ & 2.14 & $3.2072 \mathrm{e}-014$ & 3.40 & $3.7038 \mathrm{e}-014$ & 4.88 \\
\hline M_RP $(2 \mathrm{~m})$ & $2.5537 \mathrm{e}-014$ & 1.87 & $3.1282 \mathrm{e}-014$ & 2.83 & $3.6124 \mathrm{e}-014$ & 4.47 \\
\hline \hline
\end{tabular}

The complexity of the proposed new algorithms is $O\left(\mathrm{~nm}^{3}\right)$. For comparison algorithm based on the Fast Fourier Transform (FFT) for solving block circulant system with circulant blocks has complexity $O(n m \log (n m))$ but it can be implemented only when the block size of the matrix $W$ is a power of two and when all blocks of $W$ are circulant [2]. Our method does not have these restrictions. The only restriction of the applicability of our method comes from the condition of the existence of solution of matrix equation (6).

\section{References}

1. J. H. Ahlberg, E. N. Nilson, J. L. Walsh, The Theory of Splines and Their Applications, Academic Press, New York, 1967.

2. R. Chan, T. Chan, Circulant preconditioners for elliptic problems, J. Numer. Linear Algebra Appl. 1 (1992) 77-101.

3. J. C. Engwerda, On the Existence of a Positive Definite Solution of the Matrix Equation $X+A^{T} X^{-1} A=I$, Linear Algebra Appl. 194 (1993) 91-108.

4. G. Golub, C. Van Loan, Matrix Computation, The John Hopkins University Press, Baltimore, 1989.

5. B. Minchev, Some Algorithms for Solving Special Tridiagonal Block Teoplitz Linear Systems, in press J. Comp. and Appl. Math.

6. B. Mintchev, I. Ivanov, On the Computer Realization of Algorithms for Solving Specilal Block Linear Systems, Aplications of Mathemathics in Engineering and Economics'27, Heron Press, Sofia (2002) 303-313.

7. C. Mingkui, On the Solution of circulant linear systems, SIAM J. Numer. Analysis 24 (1987) 668-683.

8. W. L. Wood, Periodicity effects on the iterative solution of elliptic difference equations, SIAM J. Numer. Analysis 8 (1971) 439-464.

9. U. Zavyalov, B. Kvasov, V. Miroshnichenko, Spline Fuctions Methods (in Russian), Moscow, 1980. 